\title{
Kinetic behaviour of free lipase and mica-based immobilized lipase catalyzing the synthesis of sugar esters
}

\begin{abstract}
The utilization of natural mica as a biocatalyst support in kinetic investigations is first described in this study. The formation of lactose caprate from lactose sugar and capric acid, using free lipase (free-CRL) and lipase immobilized on nanoporous mica (NER-CRL) as a biocatalyst, was evaluated through a kinetic study. The apparent kinetic parameters, $\mathrm{K}(\mathrm{m})$ and $\mathrm{V}(\max )$, were determined by means of the Michaelis-Menten kinetic model. The PingPong Bi-Bi mechanism with single substrate inhibition was adopted as it best explains the experimental findings. The kinetic results show lower $K(\mathrm{~m})$ values with NER-CRL than with free-CRL, indicating the higher affinity of NER-CRL towards both substrates at the maximum reaction velocity $(\mathrm{V}(\max , \mathrm{app})>\mathrm{V}(\max ))$. The kinetic parameters deduced from this model were used to simulate reaction rate data which were in close agreement with the experimental values.
\end{abstract}

Keyword: Lipase immobilization; Mica; Kinetic study; Ping-Pong Bi-Bi model; Sugar ester synthesis 